\title{
Impact of Donor Age in Donation After Circulatory Death Liver Transplantation: Is the Cutoff "60" Still of Relevance?
}

\section{TO THE EDITOR:}

Schlegel et al. must be congratulated on their recent study published in Liver Transplantation that describes the wide experience of the Birmingham group with donation after circulatory death (DCD) liver transplantation (LT). ${ }^{(1)}$ In this article, the authors analyzed their results from more than 300 DCD LTs, with nearly one-third of the donors aged $>60$ years or more. They did not find a significant difference in graft loss related to DCD donor age. ${ }^{(1)}$ Donor age in DCD LT remains a challenging issue. ${ }^{(2)}$ The Schlegel study confirms our group experience with DCD donors older than 70 years, ${ }^{(3)}$ and it adds more evidence to the fact that older DCD donors might positively contribute to the liver donor pool, if other risk factors are actively minimized. ${ }^{(4,5)}$ In our clinical practice, these grafts are preferentially allocated to LT candidates aged over 50 years, undergoing a straightforward first liver transplantation that should not require high catecholamine or transfusion needs, and in whom cold ischemia and reimplantation times might be minimized. Patients suffering from hepatocellular carcinoma are ideal candidates for these older DCD grafts.

Another interesting finding of the Schlegel study is the fact that donor body mass index (BMI) might be a

Address reprint requests to Olivier Detry, M.D., Ph.D., Department of Abdominal Surgery and Transplantation, Centre Hospitalier Universitaire Liege, University of Liege, Sart Tilman B35, B4000 Liege, Belgium. Telephone: +3243667645; FAX: +3243667069; E-mail: olivier.detry@transplantation.be

Received September 15, 2017; accepted September 15, 2017.

Copyright $(2017$ by the American Association for the Study of Liver Diseases.

View this article online at wileyonlinelibrary.com.

DOI 10.1002/lt.24951

Potential conflict of interest: Nothing to report. major prognostic factor for DCD liver graft loss. ${ }^{(1)}$ In our experience with DCD kidney transplantation, donor BMI has also proven to be an important risk factor for delayed graft function. ${ }^{(6)}$ This might indicate that it is particularly difficult to rapidly lower the abdominal temperature of DCD donors with high BMI using in situ flushing and topical cooling. Further studies are needed to confirm these findings and to explore the means to improve abdominal graft results using high BMI DCD donors, considering the current and future obesity epidemic.

\author{
Olivier Detry, M.D., Ph.D. \\ Nicolas Meurisse, M.D. \\ Pierre Honoré, M.D. \\ Department of Abdominal Surgery and \\ Transplantation \\ Centre Hospitalier Universitaire Liege \\ University of Liege \\ Liege, Belgium
}

\section{REFERENCES}

1) Schlegel A, Scalera I, Thamara M, Perera PR, Kalisvaart $M$, Mergental $\mathrm{H}$, et al. Impact of donor age in donation after circulatory death liver transplantation: is the cutoff " 60 " still of relevance? Liver Transpl 2018;24:352-362.

2) Doyle MB, Collins K, Vachharajani N, Lowell JA, Shenoy $S$, Nalbantoglu I, et al. Outcomes using grafts from donors after cardiac death. J Am Coll Surg 2015;221:142-152.

3) Detry O, Deroover A, Meurisse N, Hans MF, Delwaide J, Lauwick S, et al. Donor age as a risk factor in donation after circulatory death liver transplantation in a controlled withdrawal protocol programme. Br J Surg 2014;101:784-792.

4) Firl DJ, Hashimoto K, O'Rourke C, Diago-Uso T, Fujiki M, Aucejo FN, et al. Impact of donor age in liver transplantation from donation after circulatory death donors: a decade of experience at Cleveland Clinic. Liver Transpl 2015;21:14941503.

5) Detry O. Donor age in liver transplantation: donation after circulatory death. J Am Coll Surg 2015;221:779.

6) Ledinh H, Weekers L, Bonvoisin C, Krzesinski JM, Monard J, de Roover A, et al. Results of kidney transplantation from controlled donors after cardio-circulatory death: a single center experience. Transpl Int 2012;25:201-209. 